\title{
What are the significant factors associated with adjacent segment disease in short-segment lumbar spinal fusion? A retrospective cohort analysis of cases with at least 2 years of follow-up at a single center, 2005-2015
}

\author{
Hong Yoon ${ }^{1}$, Jung-Ho Kim ${ }^{1}$, Novan Krisno Adji ${ }^{2}$, Seung-Hwan Yoon ${ }^{1}$, James-Ki Shinn ${ }^{3}$, Dal-Sung Ryu ${ }^{1}$ \\ ${ }^{1}$ Department of Neurosurgery, Inha University College of Medicine, Incheon, Korea \\ ${ }^{2}$ Neurosurgery Department, Faculty of Medicine University of Jember, Dr. Soebandi General Hospital, Jember, Indonesia \\ ${ }^{3}$ Department of Neurosurgery, Cheongju Prime Hospital, Cheongju, Korea
}

Objective: The purpose of this paper was to identify risk factors for developing adjacent segment disease (ASD) in patients who undergo short-level lumbar spinal fusion surgery through a retrospective cohort analysis focused on spinopelvic parameters.

Methods: A retrospective cohort study was conducted of 117 patients who underwent short-level lumbar spinal fusion surgery from January 2005 to December 2015. The patients were divided into 2 groups (control [non-ASD] and ASD) at a 1:1 ratio, with 28 patients per group. Spinopelvic parameters were analyzed on radiographs. Risk factors, such as Pfirrmann grade, age, and osteoporosis were also used to compare the 2 groups.

Results: No significant difference was found between the ASD group and the control group in baseline demographic characteristics. The Pfirrmann grade was higher in the ASD group $(P=0.022)$. The spinopelvic parameters with statistically significant differences between the 2 groups were lumbar lordosis (LL) and pelvic incidence (PI)-LL. The LL of the control group was higher than that of the ASD group (preoperation, $\mathrm{P}=0.022$; postoperation, $\mathrm{P}=0.012$ ). The $\mathrm{PI}-\mathrm{LL}$ value was smaller in the control group than in the ASD group (preoperation, $P=0.043$; postoperation, $P=0.042$ ).

Conclusion: LL, PI-LL, and the Pfirrmann grade were correlated with the incidence of ASD. Therefore, it is important for spinal surgeons to consider patients' image profiles related to sagittal imbalance and lumbar disc status when planning short-level lumbar spinal fusion.

Keywords: Spinal fusions; Spinopelvic parameter; Adjacent segment degeneration

\section{Introduction}

Spinal fusion has become the standard treatment for many pathological conditions of the vertebrae. This surgical procedure removes the motion that causes pain, stabilizes the spine, and allows decompression of neural elements. However, spinal fusion increases the stress on the adjacent segments, and in the long term, pa-

Received: June 3, 2021

Revised: August 10, 2021

Accepted: August 12, 2021

Corresponding Author: Seung-Hwan Yoon, MD, PhD

Department of Neurosurgery, Inha University Hospital, Inha University

College of Medicine, 27 Inhang-ro, Jung-gu, Incheon 22332, Korea

Tel: +82-32-890-2370; Fax: +82-32-890-2374;

E-mail:nsyoon@gmail.com tients are at risk for developing a common complication known as adjacent segment disease (ASD) [1].

The term "adjacent segment degeneration" is used to describe radiographic changes seen at levels adjacent to a previous spinal fusion procedure that are not necessarily correlated with any clinical findings. In contrast, the term "adjacent segment disease" is used to refer to the development of new clinical symptoms corresponding to radiographic changes adjacent to the previous level of spinal fusion [2].

There are several risk factors for ASD, including risk factors that are present before the operation, such as age, disc degeneration, facet joint degeneration, smoking, sex, osteoporosis, and physical activity. However, relatively little research has explored the role of surgical factors such as the number of fused segments, segment 
damage during surgery, fusion method, floating fusion, sagittal alignment, and spinopelvic parameters [3]. Of note, spinopelvic parameters have been widely used to describe the degree of sagittal imbalance in adult spine deformity patients. The balance between lumbar lordosis (LL) and spinopelvic parameters enables effective load absorption of the spine and effective action of the spinal muscles [4].

In patients with sagittal imbalance, correcting the sagittal alignment improves spinal biomechanical efficiency and reduces energy consumption [5]. Spinopelvic parameters are mainly used to describe sagittal imbalance or the goal of sagittal imbalance surgery. Therefore, the terminology related to spinopelvic parameters has often been used in relation to spinal deformity surgery such as long-level fusion. As the relationship between pelvic incidence (PI) as a morphological parameter and LL seems important for the sagittal profile of the spine, it may also account for different loading patterns in the lumbar spine, which may be relevant for the development of adjacent segment degeneration and disease [5-7].

Most cases of ASD occur after multi-segment fixation for spinal deformity. Various risk factors and problems during surgery are known to increase the risk of ASD after multi-segment fixation, including patient-related factors such as sex, age, body mass index, smoking, preexisting degeneration of adjacent discs, and menopause, and surgical factors such as stiffness of the implants, technical errors in the execution of fusion, and sagittal and coronal balance disturbance $[2,8,9]$. However, very few studies have explored whether ASD after short-segment fixation is related to the risk factors of ASD after multi-segment fixation, such as spinopelvic parameters. Limited research has been published have been published on the relationship between ASD after short-segment fixation and high-risk factors or spinopelvic parameters after multi-segment fixation [1].

The objective of the present study was to examine the factors that can cause ASD in short-level lumbar fusion, with the goal of enabling spinal surgeons to design procedures with appropriate consideration of risk factors. We focused on spinopelvic parameters as risk factors for ASD after short-level spinal fusion.

\section{Material and Method}

\section{Study design}

The research was conducted at a single medical center from January 2005 to December 2015. The populations in this study were patients with short-level lumbar fusion with at least 2 years of follow-up who were admitted from January 2005 to December 2015 according to the sample criteria. The Institutional Review Board (IRB) at the Inha University (IRB No. 2020-03-019) approved this study, and the informed consent requirement was waived. The data analyzed in this study came from all medical records documenting short-level lumbar fusion surgery and the occurrence of adjacent segment degeneration with follow-up of at least 2 years. Samples were obtained based on the following criteria.

The inclusion criteria in this study were 1 to 3 fusion levels, no history of previous lumbar spine surgery, the availability of preoperative and postoperative X-ray images with visible femur heads, and at least 2 years of follow-up after surgery. The exclusion criteria were surgery related to infection and surgery related to cancer metastasis.

Of the 117 patients, 28 patients with ASD were identified according to the following radiographic ASD criteria on spine X-rays. Radiographic ASD on radiographs was defined as a reduction of $\leq 50 \%$ in disc height on a neutral lateral radiograph, an increase of $\geq 3 \mathrm{~mm}$ in a vertebral slip on a neutral lateral radiograph, and a decrease of $\geq 5^{\circ}$ in the intervertebral angle on a flexion lateral radiograph [10].

Purposive sampling was used in the present study. This technique is conducted to determine the sample for a study with specific considerations in order to obtain more representative data. The number of patients who underwent short-level lumbar fusion was 117 patients, and the number of patients with ASD was 28 patients. The control and ASD groups in this study were matched at a 1:1 ratio (Fig. 1).

In this study, a predesigned instrument was used to extract information from medical records on patient identity, age, sex, level of fusion, the results of bone mineral density, the Pfirrmann grade, and the follow-up period. Mean values were obtained for the above variables and compared between the control and ASD groups. The statistical significance of differences was evaluated.

\section{Spinopelvic parameters}

The mean preoperative and postoperative values of spinopelvic

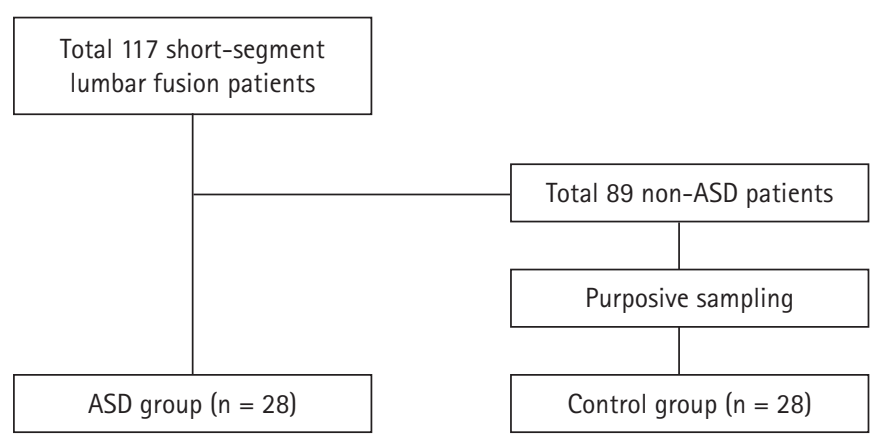

Fig. 1. Flow chart depicting patient selection. ASD, adjacent segment disease. 
parameters such as sacral slope (SS), pelvic tilt (PT), PI, LL, and PI-LL were obtained and compared between the ASD and control groups. The statistical significance of differences was assessed.

\section{Statistical analysis}

The descriptive data are presented as the mean \pm standard deviation. The chi-square test and Mann-Whitney test were performed to compare categorical variables between 2 independent groups, and P-values $<0.05$ were considered to indicate statistical significance. The normality of data distribution was assessed using the Kolmogorov-Smirnov test. Data were analyzed using SPSS version 23.0 (IBM Corp., Armonk, NY, USA).

\section{Results}

\section{Baseline characteristics}

The demographic characteristics of the total sample of 117 patients are presented in Table 1 . The baseline characteristics of both groups were compared. The sex ratio was not significantly different between the 2 groups (male:female, $10: 18$ vs. 10:18; $\mathrm{P}=0.943$ ). More than $90 \%$ of patients were older than 50 in both groups (92.9\% vs. 96.4\%). There was no significant difference in age between the 2 groups $(\mathrm{P}=0.556)$. Osteoporosis patients were more common in the ASD group, but this difference was not statistically significant ( $14.3 \%$ vs. $28.6 \%, \mathrm{P}=0.329)$. There were significantly fewer levels of fusion in the control group than in the ASD group $(\mathrm{P}=0.040)$. The average follow-up period of the 2 groups was less than 5 years, and there was no significant difference in this regard between the 2 groups $(\mathrm{P}=0.471)$ (Table 1 ).

Of the 28 ASD patients, 10 were men and 18 were women. The 11 patients who underwent repair surgery comprised 2 male patients and 9 female patients. The overwhelming majority of patients who underwent lumbar fusion surgery were older than 50 years of age $(n=111)$. Among the 28 ASD patients, the location of the ASD was only distal in 2 patients.

\section{Pfirrmann grade}

The Pfirrmann grading system evaluates degenerated discs by T2-weighted magnetic resonance imaging according to the disc structure asymmetry, distinction between nucleus and the annulus, signal intensity of discs, and the height of discs; on this basis, a grade from 1 to 5 is assigned for disc degeneration. Grade 1 is defined as a homogeneous, bright white disc with normal disc height. Grade 2 is defined as an inhomogeneous white disc with a clear distinction from the annulus fibrosus and normal disc height. Grade 3 is defined as an inhomogeneous gray disc with an unclear distinction from the annulus fibrosus and normal disc height. Grade 4 is defined as an inhomogeneous gray-to-black disc without distinction from the annulus fibrosus. Grade 5 is defined as an inhomogeneous black disc with a collapsed intervertebral space [11].

Table 1. Baseline characteristics of the control and ASD groups

\begin{tabular}{|c|c|c|c|c|}
\hline Variable & Control group $(n=28)$ & ASD group $(n=28)$ & Total $(n=117)$ & P-value \\
\hline Sex (male:female) & $10: 18$ & $10: 18$ & $42: 75$ & 0.943 \\
\hline Age (yr) & & & & 0.556 \\
\hline$<50$ & $2(7.1)$ & 1 (3.6) & $6(5.1)$ & \\
\hline$\geq 50$ & $26(92.9)$ & 27 (96.4) & $111(94.9)$ & \\
\hline Osteoporosis & $4(14.3)$ & $8(28.6)$ & $21(17.9)$ & 0.329 \\
\hline Level of fusion & & & & $0.040^{*}$ \\
\hline 1 & $14(50.0)$ & $11(39.3)$ & $53(45.3)$ & \\
\hline 2 & $13(46.4)$ & 8 (28.6) & $46(39.3)$ & \\
\hline 3 & 1 (3.6) & $9(32.1)$ & 18 (15.4) & \\
\hline Follow-up (yr) & & & & 0.471 \\
\hline$<5$ & $22(78.6)$ & 26 (92.9) & 108 (92.3) & \\
\hline$\geq 5$ & $6(21.4)$ & $2(7.1)$ & $9(7.7)$ & \\
\hline Pfirrmann grade & & & & $0.022^{*}$ \\
\hline 2 & $1(3.6)$ & 0 & $1(0.9)$ & \\
\hline 3 & $16(57.1)$ & $7(25.0)$ & 49 (41.9) & \\
\hline 4 & $7(25.0)$ & $16(57.1)$ & $48(41.0)$ & \\
\hline 5 & $4(14.3)$ & $5(17.9)$ & $19(16.2)$ & \\
\hline
\end{tabular}

Values are presented as number (\%). $A S D$, adjacent segment disease. ${ }^{*} \mathrm{P}<0.05$, statistically significant. 
The distribution of Pfirrmann grades was as follows in the control group: grade 2, 1 patient; grade 3, 16 patients; grade 4, 7 patients; and grade 5, 4 patients. The corresponding distribution in the ASD group was as follows: grade 2, 0 patients; grade 3, 7 patients; grade 4, 16 patients; and grade 5, 5 patients. The control group and the ASD group showed a statistically significant difference in the Pfirrmann grade $(\mathrm{P}=0.022)$ (Table 1$)$.

\section{Spinopelvic parameters}

The spinopelvic parameters used to determine the risk factors of ASD were preoperative and postoperative values of SS, PT, PI, LL, and PI-LL (Table 2, Fig. 2). The mean preoperative SS of the control group was similar to that of the ASD group $\left(30.20^{\circ} \pm 8.08^{\circ} \mathrm{vs}\right.$. $\left.29.77^{\circ} \pm 8.38^{\circ}\right)$. This difference was not statistically significant $(\mathrm{P}=0.695)$. The mean postoperative SS was similar in both groups; likewise, the difference was not statistically significant $\left(29.71^{\circ} \pm 5.57^{\circ}\right.$ vs. $\left.27.61^{\circ} \pm 8.10^{\circ}, \mathrm{P}=0.346\right)$.

The mean preoperative PT was lower in the ASD group than in the control group $\left(17.27^{\circ} \pm 12.47^{\circ}\right.$ vs. $\left.24.15^{\circ} \pm 10.40^{\circ}\right)$, but the difference was not statistically significant $(P=0.723)$. The mean postoperative $\mathrm{PT}$ was higher in the ASD group than in the control group $\left(15.55^{\circ} \pm 9.59^{\circ}\right.$ vs. $\left.25.79^{\circ} \pm 9.35^{\circ}\right)$; however, this difference did not show statistical significance $(\mathrm{P}=0.864)$.

The mean preoperative and postoperative PI values of the control group were slightly lower than those of the ASD group (preoperation, $47.47^{\circ} \pm 13.52^{\circ}$ vs. $53.92^{\circ} \pm 11.01^{\circ}$; postoperation, $45.26^{\circ} \pm$ $9.75^{\circ}$ vs. $\left.53.41^{\circ} \pm 10.54^{\circ}\right)$. Neither difference was statistically significant (preoperation, $\mathrm{P}=0.186$; postoperation, $\mathrm{P}=0.357$ ).

However, the spinopelvic parameter data showed significant differences in LL and PI-LL before and after short-level lumbar fusion (preoperative $\mathrm{LL}, \mathrm{P}=0.022$; postoperative $\mathrm{LL}, \mathrm{P}=0.012$; preoperative PI-LL, $\mathrm{P}=0.043$; postoperative $\mathrm{PI}-\mathrm{LL}, \mathrm{P}=0.042$ ). The control group had higher mean preoperative and postoperative $\mathrm{LL}$ than the ASD group (preoperation, $34.68^{\circ} \pm 10.36^{\circ}$ vs. $27.70^{\circ} \pm$ $12.82^{\circ}$; postoperation, $40.15^{\circ} \pm 8.50^{\circ}$ vs. $27.83^{\circ} \pm 11.56^{\circ}$ ), as well as lower preoperative and postoperative PI-LL (preoperation, $12.79^{\circ} \pm 11.19^{\circ}$ vs. $26.22^{\circ} \pm 11.41^{\circ}$; postoperation, $5.12^{\circ} \pm 5.00^{\circ}$ vs. $\left.25.58^{\circ} \pm 12.52^{\circ}\right)$.

\section{Discussion}

In this study, the risk factors for ASD in short-segment lumbar fusion were established by comparing the control group and the ASD group. The Pfirrmann grade and spinopelvic parameters such as LL and PI-LL were found to be statistically significant
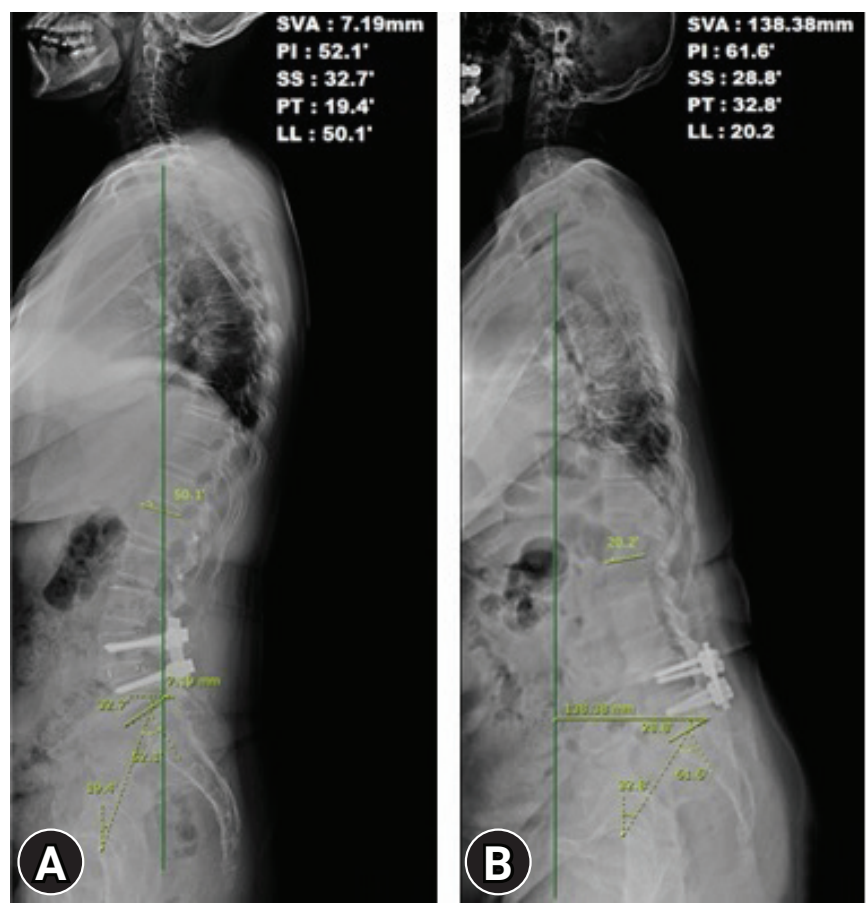

Fig. 2. (A) and (B) are lateral views of the lumbar spine and radiologic measurements at 2 years of follow-up after surgery. $(A)$ is an example of the control group and $(B)$ is a sample from the adjacent segment disease group. SVA, sagittal vertical axis; PI, pelvic incidence; SS, sacral slope; PT, pelvic tilt; LL, lumbar lordosis.

Table 2. Preoperative and postoperative values of spinopelvic parameters

\begin{tabular}{|c|c|c|c|c|c|c|}
\hline \multirow{2}{*}{ Spinopelvic parameter $\left({ }^{\circ}\right)$} & \multicolumn{3}{|c|}{ Preoperative } & \multicolumn{3}{|c|}{ Postoperative } \\
\hline & Control group & ASD group & P-value & Control group & ASD group & P-value \\
\hline Sacral slope & $30.20 \pm 8.08$ & $29.77 \pm 8.38$ & 0.695 & $29.71 \pm 5.57$ & $27.61 \pm 8.10$ & 0.346 \\
\hline Pelvic tilt & $17.27 \pm 12.47$ & $24.15 \pm 10.40$ & 0.723 & $15.55 \pm 9.59$ & $25.79 \pm 9.35$ & 0.864 \\
\hline Pelvic incidence & $47.47 \pm 13.52$ & $53.92 \pm 11.01$ & 0.186 & $45.26 \pm 9.75$ & $53.41 \pm 10.54$ & 0.357 \\
\hline Lumbar lordosis & $34.68 \pm 10.36$ & $27.70 \pm 12.82$ & $0.022^{*}$ & $40.15 \pm 8.50$ & $27.83 \pm 11.56$ & $0.012^{*}$ \\
\hline Pelvic incidence-lumbar lordosis & $12.79 \pm 11.19$ & $26.22 \pm 11.41$ & $0.043^{*}$ & $5.12 \pm 5.00$ & $25.58 \pm 12.52$ & $0.042^{*}$ \\
\hline
\end{tabular}

Values are presented as the mean \pm standard deviation.

ASD, adjacent segment disease.

${ }^{*} \mathrm{P}<0.05$, statistically significant. 
risk factors.

According to Masevnin et al. [12] in 2016, a Pfirrmann grade of 3 or more is associated with a high risk for ASD in short-segment lumbar fusion. In this study, the Pfirrmann grade showed a significant difference between the control and ASD groups. Lower Pfirrmann grades are more susceptible to stress and segment degeneration. This phenomenon was also seen in patients with proximal junctional kyphosis who developed ASD after long-segment spinal fusion [13].

No significant difference in mean age was found between the control group and ASD group. This shows that age is not an important factor regarding the risk of ASD in short-segment lumbar fusion. However, the results of this study contradict those reported by Cho et al. [14] in 2009, according to which an age of more than 50 years is associated with an elevated risk of ASD.

No significant difference was found in bone mineral density between the ASD group and the control group, which suggests that osteoporosis is not a risk factor for ASD. The results of this study contrast in this regard with the research conducted by $\mathrm{Ha}$ et al. [15] and Wang et al. [9].

It has been suggested that spinopelvic parameters may contribute to adjacent segment degeneration. Kumar et al. [16] in 2001 concluded that patients with a normal postoperative $\mathrm{C} 7$ plumbline and sacral inclination had the lowest risk of adjacent segment degeneration. However, the relationships of adjacent segment degeneration after lumbar fusion with spinopelvic parameters, such as LL, PI, PT, and SS, have never been described. Legaye et al. in 1998 [4] and Duval-Beaupère et al. in 1992 [17] described a chain of correlations between the positional parameters of upright posture, and PI. Boulay et al. [6] in 2006 demonstrated an equation for predicting LL based on PI and explained that within the confidence limits, the standing position is within the conditions of an economic posture, whereas out of the confidence limits, the adaptation potential of the spine and pelvis is exceeded, potentially evoking pathological positions and loading patterns. PI has subsequently been generally acknowledged as a predictor of the amount of LL required to assume a balanced sagittal posture $[5,7,17]$.

The LL angle was significantly lower in the ASD group than in the control group, whereas the mean PI-LL value of the control group was significantly lower than that of the ASD group. This is similar to the finding of a study by Rothenfluh et al. [18] in 2015, according to which a PI-LL value greater than $10^{\circ}$ can be a cause of ASD after lumbar spinal fusion. Masevnin et al. [8] in 2015 stated that abnormal spinopelvic parameters were associated with a high risk of ASD after lumbar spinal fusion. However, SS, PT, and PI did not show a significant difference after short-level lumbar spinal fusion in this study.

It can be seen that ASD can be prevented by suitable lordotic curves in short-level lumbar fusion. In other words, it is important to make a lordotic curve by carefully selecting the size or type of cage during short-level lumbar fusion. In addition, when planning surgery, creating a design that reduces the PI-LL value is an important point to consider for reducing the risk of ASD.

The lumbar spine is compensated by minimizing the mismatch between PI and LL values [19]. In general, LL has been found to be very important in maintaining an effective standing posture. When the lumbar lordotic curve becomes smaller after surgery, an attempt is made to create $\mathrm{LL}$ in the remaining area. In short-level lumbar fusion, as investigated in this study, the lumbar spine tends to compensate by performing an extension motion at the upper and lower level of the fusion site. Before surgery, the lumbar spine generally rotates around L5/S1 with a pivot, but when surgery is performed, a fixed segment is rotated with a pivot. As a whole, in order to have the same momentum as before surgery, the length of the lever arm is shorter in the adjacent joint, so a greater force is required, which induces segmental regression and is thought to cause ASD. In addition, the upper segment requires more force because it has a larger moment of inertia since it includes the head, trunk, and arms. In this regard, biomechanical research is needed in the future.

The PT is a dynamic pelvic index that changes according to the rotation of the pelvis [20]. The change in its value is the most important index to determine the pelvic balance or the degree of pelvic compensation in a pathological condition. PT increases as part of the compensatory action for decreasing LL and increasing thoracic kyphosis with aging [21]. In this study, the postoperative PT value of the ASD group was $25.79^{\circ} \pm 9.35^{\circ}$, which was considerably greater than the postoperative PT value of the control group $\left(15.55^{\circ} \pm 9.59^{\circ}\right)$. Although this difference did not show statistical significance, it would appear that a higher after short-level lumbar spinal fusion may be associated with a higher incidence of ASD.

This study had several limitations. In the comparison between the ASD group and the control group, the baseline variables with a P-value of less than 0.05 were Pfirrmann grade, levels of fusion, LL, and PI-LL. Therefore, the first limitation is that it is not clear whether the risk of ASD is due to the difference in LL and PI-LL, disc degeneration, or levels of fusion. The next limitation is this research had a retrospective design with a relatively small sample size. In addition, since this study was conducted at a single institution, the results might have been affected by the distinct characteristics of the area. A multi-center study with a larger number of patients from diverse regions will be needed to decrease the possibility of bias. 


\section{Conclusion}

As spinopelvic parameters, LL and PI-LL were found to be correlated with ASD incidence. Age and osteoporosis-related parameters were not associated with the incidence of ASD, whereas the Pfirrmann grade was associated with the risk of ASD. In conclusion, to prevent ASD after short-level lumbar spinal fusion, spine surgeons should consider appropriate lumbar lordotic curve planning before the operation and decide carefully whether lumbar levels with severe disc degeneration should be included in the surgical range.

\section{Conflicts of interest}

No potential conflict of interest relevant to this article was reported.

\section{Acknowledgments}

This work was supported by an Inha University Hospital Research Grant.

\section{ORCID}

Hong Yoon, https://orcid.org/0000-0001-5250-9747

Jung-Ho Kim, https://orcid.org/0000-0001-6067-8823

Novan Krisno Adji, https://orcid.org/0000-0002-2914-5970

Seung-Hwan Yoon, https://orcid.org/0000-0003-0558-2313

James-Ki Shinn, https://orcid.org/0000-0002-4994-2926

Dal-Sung Ryu, https://orcid.org/0000-0003-0895-3431

\section{REFERENCES}

1. Scemama C, Magrino B, Gillet P, Guigui P. Risk of adjacent-segment disease requiring surgery after short lumbar fusion: results of the French Spine Surgery Society Series. J Neurosurg Spine 2016;25:46-51.

2. Hilibrand AS, Robbins M. Adjacent segment degeneration and adjacent segment disease: the consequences of spinal fusion? Spine J 2004;4(6 Suppl):190S-194S.

3. Lee JC, Choi SW. Adjacent segment pathology after lumbar spinal fusion. Asian Spine J 2015;9:807-17.

4. Legaye J, Duval-Beaupère G, Hecquet J, Marty C. Pelvic incidence: a fundamental pelvic parameter for three-dimensional regulation of spinal sagittal curves. Eur Spine J 1998;7:99-103.

5. Schwab F, Patel A, Ungar B, Farcy JP, Lafage V. Adult spinal deformity-postoperative standing imbalance: how much can you tolerate? An overview of key parameters in assessing alignment and planning corrective surgery. Spine (Phila Pa 1976) 2010;35: 2224-31.

6. Boulay C, Tardieu C, Hecquet J, et al. Sagittal alignment of spine and pelvis regulated by pelvic incidence: standard values and prediction of lordosis. Eur Spine J 2006; 15:415-22.

7. Roussouly P, Gollogly S, Berthonnaud E, Dimnet J. Classification of the normal variation in the sagittal alignment of the human lumbar spine and pelvis in the standing position. Spine (Phila Pa 1976) 2005;30:346-53.

8. Masevnin S, Ptashnikov D, Michaylov D, Meng H, Smekalenkov $\mathrm{O}$, Zaborovskii N. Risk factors for adjacent segment disease development after lumbar fusion. Asian Spine J 2015;9:239-44.

9. Wang H, Ma L, Yang D, et al. Incidence and risk factors of adjacent segment disease following posterior decompression and instrumented fusion for degenerative lumbar disorders. Medicine (Baltimore) 2017;96:e6032.

10. Nakashima H, Kawakami N, Tsuji T, et al. Adjacent segment disease after posterior lumbar interbody fusion: based on cases with a minimum of 10 years of follow-up. Spine (Phila Pa 1976) 2015;40:E831-41.

11. Rim DC. Quantitative Pfirrmann disc degeneration grading system to overcome the limitation of Pfirrmann disc degeneration grade. Korean J Spine 2016;13:1-8.

12. Masevnin S, Ptashnikov D, Mikhaylov D, Smekalenkov O, Zaborovskii N, Lapaeva O. Early adjacent segment degeneration after short lumbar fusion. Global Spine J 2016;6(1_suppl): s-0036-1582968.

13. Glattes RC, Bridwell KH, Lenke LG, Kim YJ, Rinella A, Edwards C 2nd. Proximal junctional kyphosis in adult spinal deformity following long instrumented posterior spinal fusion: incidence, outcomes, and risk factor analysis. Spine (Phila Pa 1976) 2005; 30:1643-9.

14. Cho KS, Kang SG, Yoo DS, Huh PW, Kim DS, Lee SB. Risk factors and surgical treatment for symptomatic adjacent segment degeneration after lumbar spine fusion.J Korean Neurosurg Soc 2009;46:425-30.

15. Ha KY, Son JM, Im JH, Oh IS. Risk factors for adjacent segment degeneration after surgical correction of degenerative lumbar scoliosis. Indian J Orthop 2013;47:346-51.

16. Kumar MN, Baklanov A, Chopin D. Correlation between sagittal plane changes and adjacent segment degeneration following lumbar spine fusion. Eur Spine J 2001;10:314-9.

17. Duval-Beaupère G, Schmidt C, Cosson P. A Barycentremetric study of the sagittal shape of spine and pelvis: the conditions required for an economic standing position. Ann Biomed Eng 1992;20:451-62.

18. Rothenfluh DA, Mueller DA, Rothenfluh E, Min K. Pelvic inci- 
dence-lumbar lordosis mismatch predisposes to adjacent segment disease after lumbar spinal fusion. Eur Spine J 2015;24: 1251-8.

19. Merrill RK, Kim JS, Leven DM, Kim JH, Cho SK. Beyond pelvic incidence-lumbar lordosis mismatch: the importance of assessing the entire spine to achieve global sagittal alignment. Global Spine J 2017;7:536-42.
20. Le Huec JC, Aunoble S, Philippe L, Nicolas P. Pelvic parameters: origin and significance. Eur Spine J 2011;20 Suppl 5(Suppl 5): 564-71.

21. Barrey C, Roussouly P, Le Huec JC, D'Acunzi G, Perrin G. Compensatory mechanisms contributing to keep the sagittal balance of the spine. Eur Spine J 2013;22 Suppl 6(Suppl 6):S834-41. 